University of Zurich

Department of Economics

Working Paper Series

ISSN 1664-7041 (print)

ISSN 1664-705X (online)

Working Paper No. 129

On the Equivalence Between Bayesian and Dominant Strategy Implementation: The Case of Correlated Types

Alexey Kushnir

August 2013 


\title{
On the Equivalence Between Bayesian and Dominant StRategy Implementation: The Case of Correlated Types
}

\author{
Alexey Kushnir*
}

July 28,2013

\begin{abstract}
We consider general social choice environments with private values and correlated types. Each agent's matrix of conditional probabilities satisfies the full rank condition. We show that for any Bayesian incentive compatible mechanism there exists a dominant strategy incentive compatible mechanism that delivers the same interim expected utilities to all agents and generates at least the same social surplus. In addition, if there is a social alternative that is inferior to the other alternatives for all agents the dominant strategy incentive compatible mechanism matches exactly the social surplus. These results extend to environments with interdependent values satisfying the single crossing condition.
\end{abstract}

JEL classifications: $D 82$

Keywords: mechanism design, Bayesian implementation, dominant strategy implementation, full surplus extraction, correlation

${ }^{*}$ Chair for Organizational Design, ESEI Center for Market Design, University of Zürich, Blümlisalpstrasse 10, Zurich, 8006, Switzerland, alexey.kushnir@esei.ch, tel.: +41 44 6345562. I gratefully acknowledge financial support from the European Research Council (ERC Advanced Investigator Grant, ESEI-249433). I thank Jacob Goeree, Robertas Zubrikas, Konrad Mierendorff, and Philippos Louis for discussions and useful suggestions. 


\section{Introduction}

The design of robust mechanisms that do not rely on the assumptions of a common prior and equilibrium play has recently attracted a lot of attention (e.g. Bergemann and Morris, 2012). The restrictions to robust mechanisms, however, can potentially limit the set of outcomes that the designer can achieve. In this paper we analyze whether for a given Bayesian incentive compatible (BIC) mechanism there exists an equivalent dominant strategy incentive compatible (DIC) mechanism that delivers the same interim expected utilities to all agents and generates the same social surplus.

The notion of mechanism equivalence based on agent interim expected utilities is introduced by Manelli and Vincent (2010) who show for standard single-unit auctions that for any BIC mechanism there exists a DIC mechanism that delivers the same interim expected utilities to all agents. Gershkov et al. (2013) require additionally the mechanisms to generate the same social surplus and extend the BIC-DIC equivalence to social choice problems with independent, one-dimensional, private types and linear utilities (see also Goeree and Kushnir, 2012).

We further extend the equivalence to general social choice problems with private values and discrete correlated types. We assume that each agent's matrix of conditional probabilities satisfies the full rank condition and show that for any BIC mechanism there exists a DIC mechanism that delivers the same interim expected utilities to all agents and generates at least the same social surplus. Moreover, if there exists a social alternative that is inferior to all other alternatives for all agents an equivalent DIC always exists. We further explain how our results apply to environments with interdependent values that satisfy the single crossing condition.

To provide intuition for our results note that the VCG mechanisms implement the maximum social surplus if agents have private values. Therefore, there exists a DIC mechanism that generates the highest possible social surplus. We then use the techniques of Cremer and McLean (1985, 1988) to tailor the VCG payments such that agent interim expected utilities match exactly the agent interim expected utilities of a given BIC mechanism.

Cremer and McLean (1985, 1988) establish that a mechanism designer can fully extract the social surplus in a classical auction model with correlated discrete types. McAfee and Reny (1992) subsequently extend their result to general mechanism design environments with continuous types. ${ }^{1}$ They state also provocatively that "... the results (full rent extraction) cast doubt on the value of the current mechanism design paradigm as a model of institutional design." This critique put forward an investigation whether the full surplus extraction result is generic. Heifetz and Neeman (2006) are first to point out that the essential deficiency of Cremer and McLean's model is the common-knowledge assumption on fixed finite number of types. ${ }^{2}$ They show that the collection of priors that permits the full surplus extraction is "small" in both a geometric sense (i.e. they are contained in a proper face) and a measure-theoretic sense

\footnotetext{
${ }^{1}$ See also McAfee et al. (1989) for the analysis of the full surplus extraction in the common-value auction with continuous types.

${ }^{2}$ The full surplus extraction result demands also other important modeling assumptions: risk neutrality, unlimited liability, absence of collusion among agents, and lack of competition among sellers (see Robert, 1991; Laffont and Marimort, 2000; Che and Kim, 2006; and Peters, 2001). Papadimitriou and Pierrakos (2011) and Csapo and Müller (2012) analyze algorithmic complexity of finding profit-maximizing mechanisms in environments with correlated types.
} 
(i.e. they are contained in a finitely shy set, as defined by Anderson and Zame (2001)). ${ }^{3}$ Chen and Xiong (2013) reexamine the question whether the full surplus extraction is generic. They relax the assumption of the full surplus extraction and show that the set of priors, for which there is the full surplus extraction except for a quantity less than $\epsilon>0$, is open and dense in the universal type space endowed with the standard weak* topology. Their conclusion suggests that our results are also generic in topological sense.

We finally acknowledge an important paper by Mookherjee and Reichelstein (1992) who are the first to study the relationship between Bayesian and dominant strategy implementation that preserves the same agents interim expected utilities. In particular, they show that for any monotone $\mathrm{BIC}$ allocation it is possible to find payments that implement this allocation in dominant strategies and leave every agent's interim expected utility unchanged. Though their analysis is limited to independent priors they also observe that "With non-independent beliefs, equivalent dominant strategy implementation of Bayesian mechanisms is still possible..." Our results, however, conceptually differ from Mookherjee and Reichelstein (1992) because we study the equivalence between mechanisms rather than the dominant strategy implementation of BIC allocation rules.

The paper is organized as follows. The model and definitions are introduced in Section 2. We illustrate our main findings with a simple example in Section 3. Our main results are presented in Section 4. Section 5 discusses how our analysis applies to the environments with interdependent values and can be extended to continuous type spaces.

\section{Model}

We consider a model with a set $\mathcal{I}=\{1, \ldots, I\}$ of agents and a set $\mathcal{K}=\{1, \ldots, K\}$ of social alternatives. Each agent has a private type $x_{i}$ taken from some finite set $X_{i}=\left\{x_{i}^{1}, \ldots, x_{i}^{N_{i}}\right\}{ }^{4}$ Let $X=\times_{i \in \mathcal{I}} X_{i}$ and $X_{-i}=\times_{j \neq i} X_{j}$. Agent types are distributed according to distribution $f$. We denote the probability of other agent types $x_{-i}$ given that agent $i$ has type $x_{i}$ as $f\left(x_{-i} \mid x_{i}\right)$. We assume that distribution $f$ satisfies the spanning condition of Cremer and McLean (1985): for all $i \in \mathcal{I}$ there do not exist $\left\{\rho_{i}\left(x_{i}\right)\right\}_{x_{i} \in X_{i}}$, not all equal to zero, such that

$$
\sum_{x_{i} \in X_{i}} \rho_{i}\left(x_{i}\right) f\left(x_{-i} \mid x_{i}\right)=0 \text { for all } x_{-i} \in X_{-i}
$$

This condition says that for each agent the matrix of conditional probabilities has full rank. Agent's value for alternative $k$ is given by $v_{i}^{k}\left(x_{i}\right)$ for some function $v_{i}^{k}: X_{i} \rightarrow R$.

We analyze direct mechanisms defined by allocation $\left\{q^{k}(\mathbf{x})\right\}_{k \in K}$ and transfers $\left\{t_{i}(\mathbf{x})\right\}_{i \in \mathcal{I}}$ where $\mathbf{x}=\left(x_{1}, \ldots, x_{I}\right)$ is a profile of agent reports. Allocation $q^{k}(\mathbf{x}) \geq 0$ specifies the probability that alternative $k$ is chosen such that $\sum_{k \in \mathcal{K}} q^{k}(\mathbf{x})=1$ and $t_{i}(\mathbf{x})$ specifies the monetary transfer to agent $i$. Therefore, agent's utility from reporting $x_{i}^{\prime}$ given his true type $x_{i}$ equals $u_{i}\left(x_{i}^{\prime}, x_{i}, x_{-i}\right)=\sum_{k \in \mathcal{K}} q_{i}^{k}\left(x_{i}^{\prime}, x_{-i}\right) v_{i}^{k}\left(x_{i}\right)+t_{i}\left(x_{i}^{\prime}, x_{-i}\right)$. We also denote agent's interim expected

\footnotetext{
${ }^{3}$ See Barelli (2009), Gizatulina and Hellwig $(2010,2012)$ for further qualifications and extensions of Heifetz and Neeman (2006)'s result.

${ }^{4}$ Agents types can be multidimensional. We also discuss how our results apply to the environments with interdependent values and continuous types in Section 5.
} 
utility as $U_{i}\left(x_{i}^{\prime} \mid x_{i}\right)=E_{x_{-i} \mid x_{i}} u_{i}\left(x_{i}^{\prime}, x_{i}, x_{-i}\right)$. The social surplus can be calculated as

$$
S=\sum_{\mathbf{x} \in \mathbf{X}} f(\mathbf{x})\left(\sum_{i \in \mathcal{I}} \sum_{k \in \mathcal{K}} q^{k}(\mathbf{x}) v_{i}^{k}\left(x_{i}\right)\right)
$$

We analyze Bayesian and dominant strategy incentive compatible mechanisms defined as follows. A direct mechanism $(\mathbf{q}, \mathbf{t})$ is Bayesian incentive compatible (BIC) if $U_{i}\left(x_{i} \mid x_{i}\right) \geq U_{i}\left(x_{i}^{\prime} \mid x_{i}\right)$ for any $x_{i}^{\prime}, x_{i}$. A direct mechanism $(\mathbf{q}, \mathbf{t})$ is dominant strategy incentive compatible (DIC) if $u_{i}\left(x_{i}, x_{i}, x_{-i}\right) \geq u_{i}\left(x_{i}^{\prime}, x_{i}, x_{-i}\right)$ for any $x_{i}^{\prime}, x_{i}$ and $x_{-i}$. We use the notion of mechanism equivalence as stated in Gershkov et al. (2013).

Definition. Two mechanisms are equivalent if and only if they deliver the same interim expected utilities for all agents and generate the same social surplus.

\section{A Simple Example}

We illustrate our results with a simple single-unit auction with two agents $i=1,2$ and two types $x^{1}<x^{2}$. The distribution of agent types is correlated and can be represented with the following matrix

$$
f=\left(\begin{array}{cc}
\frac{3}{8} & \frac{1}{8} \\
\frac{1}{8} & \frac{3}{8}
\end{array}\right)
$$

where the rows correspond to agent 1's type and the columns to agent 2's type, and the entries correspond to the probability that vector of types $\left(x^{1}, x^{2}\right)$ is realized. It is straightforward to check the matrix of conditional probabilities satisfies spanning condition (1).

Let us consider some BIC mechanism $(\tilde{\mathbf{q}}, \tilde{\mathbf{t}})$ that delivers interim expected utilities $\widetilde{U}_{i}\left(x_{i}\right)$ to agents and generates social surplus $\widetilde{S}$. We now construct a DIC mechanism that delivers the same interim expected utilities to all agents and generates at least the same social surplus.

We consider the second-price auction with the efficient allocation rule and transfers

$$
\mathbf{q}^{*}=\left(\begin{array}{cc}
\frac{1}{2} & 0 \\
1 & \frac{1}{2}
\end{array}\right) \quad \mathbf{t}^{*}=\left(\begin{array}{cc}
-\frac{1}{2} x^{1} & 0 \\
-x^{1} & -\frac{1}{2} x^{2}
\end{array}\right)
$$

where the rows correspond to agent 1's type and the columns to agent 2's type, and the entries correspond to the probabilities that agent 1 gets the object and receives the corresponding transfers. The allocation and transfers for agent 2 are constructed symmetrically. The secondprice auction $\left(\mathbf{q}^{*}, \mathbf{t}^{*}\right)$ is DIC and generates the highest possible social surplus $S^{*} \geq \widetilde{S}$. Let us consider agent $i$ and denote

$$
h^{j}=\widetilde{U}_{i}\left(x^{j}\right)-U_{i}^{*}\left(x^{j}\right)
$$


for $j=1,2$. Consider $g^{j}$ such that

$$
g^{1}=\frac{3}{2} h^{1}-\frac{1}{2} h^{2} \quad g^{2}=\frac{3}{2} h^{2}-\frac{1}{2} h^{1}
$$

Note that the above functions satisfy that $f\left(x^{1} \mid x^{j}\right) g^{1}+f\left(x^{2} \mid x^{j}\right) g^{2}=h^{j}$ for each $j=1,2$ (the existence of $g^{j}$ for any given $h^{j}$ is guaranteed by the spanning condition (1)). We now consider modified transfers $t_{i}\left(x_{i}, x^{j}\right)=t_{i}^{*}\left(x_{i}, x^{j}\right)+g^{j}, j=1,2$. Since the additional term for agent $i$ depends only on the other agent's type it does not change agent $i$ 's incentives. At the same time the expected interim utility in the constructed mechanism $\left(\mathbf{q}^{*}, \mathbf{t}\right)$ matches exactly the interim expected utility of agents in BIC mechanism $(\tilde{\mathbf{q}}, \tilde{\mathbf{t}})$ and generates at least the same social surplus.

\section{Results}

In this section we present our main results that consist of two theorems and an example. Following the logic of the example in Section 3, Theorem 1 constructs for any BIC mechanism a DIC mechanism that delivers the same interim expected utilities to all agents and generates at least the same surplus. Theorem 2 provides a sufficient condition when the social surplus of some DIC mechanism can exactly match the social surplus of any given BIC mechanism. Finally, the example illustrates why one cannot always match the social surplus exactly.

Theorem 1. For any BIC there exists a DIC mechanism that delivers the same interim expected utility to all agents and generates at least the same social surplus.

Proof. Let us consider some BIC mechanism $(\tilde{\mathbf{q}}, \tilde{\mathbf{t}})$ that delivers interim expected utility $\widetilde{U}_{i}\left(x_{i}\right)$ to agent $i$ with type $x_{i}$ and generates social surplus $\widetilde{S}$. We denote $\left(\mathbf{q}^{*}, \mathbf{t}^{*}\right)$ be the pivot VCG mechanism (see Chapter 2 in Milgrom, 2004). The pivot VCG mechanism generates the highest possible social surplus $S^{*}$ and satisfies DIC constraints. Let $U_{i}^{*}\left(x_{i}\right)$ be the interim expected utility of agent $i$ with type $x_{i}$ from participating in the pivot VCG mechanism. Let us denote

$$
h_{i}\left(x_{i}\right)=\widetilde{U}_{i}\left(x_{i}\right)-U_{i}^{*}\left(x_{i}\right)
$$

for each $x_{i}$ and $i \in \mathcal{I}$. Given that the distribution function satisfies spanning condition (1) we employ the construction of Cremer and McLean (1985) to find $\left\{g_{i}\left(x_{-i}\right)\right\}_{x_{-i} \in X_{-i}}$ such that

$$
\sum_{x_{-i} \in X_{-i}} f\left(x_{-i} \mid x_{i}\right) g_{i}\left(x_{-i}\right)=h_{i}\left(x_{i}\right)
$$

We now consider transfers $t_{i}(\mathbf{x})=t_{i}^{*}(\mathbf{x})+g_{i}\left(x_{-i}\right)$. Since the additional term depends only on the types of the other agents the modified transfers still satisfy DIC constraints for the allocation rule of pivot VCG mechanism $\mathbf{q}^{*}$. At the same time the expected utility in the constructed mechanism $\left(\mathbf{q}^{*}, \mathbf{t}\right)$ matches exactly the expected utility of the given BIC mechanism $(\tilde{\mathbf{q}}, \tilde{\mathbf{t}})$. 
Remark 1. The constructed DIC mechanism is not necessarily ex post individually rational. Moreover, we cannot generally demand both a stronger notion of incentive compatibility and individual rationality to extract the full social surplus and, hence, match agent interim utilities in the environments with correlated types, e.g. Csapo and Müller (2012). This is in contrast to the previous BIC-DIC equivalence results for independently distributed types. In particular, Gershkov et al. (2013) and Goeree and Kushnir (2012) show that for any BIC and interim individually rational mechanism there exist an equivalent DIC and ex post individually rational mechanism when agents have independent, one-dimensional types, private values, and linear utilities.

Remark 2. Gershkov et al. (2013) refer to an example due to Cremer and Mclean (1988) that shows that the mechanism designer can extract the full social surplus with a BIC mechanism, but not with a DIC mechanism, when spanning condition (1) is violated. This example also reaffirms that condition (1) is essential for our results.

Let us now consider environments in which there exists a social alternative $k_{0}$ that delivers the smallest utility to all agents independently on their types, e.g. not allocating the object in auction settings. To normalize we assume that $v_{i}^{k_{0}}\left(x_{i}\right) \equiv 0$ and $v_{i}^{k}\left(x_{i}\right) \geq 0$ for each $i \in \mathcal{I}$, $k \in \mathcal{K}$ and $x_{i} \in X_{i}$.

Theorem 2. If there exists a social alternative that is inferior to all other alternatives for all agents independently of their types, then for any BIC mechanism there exist an equivalent DIC mechanism.

Proof. Let us consider some BIC mechanism $(\tilde{\mathbf{q}}, \tilde{\mathbf{t}})$ that generates social surplus $\widetilde{S}$. Let us pick the pivot VCG mechanism $\left(\mathbf{q}^{*}, \mathbf{t}^{*}\right)$. This mechanism generates the highest possible social surplus $S^{*} \geq \widetilde{S}$ and satisfies DIC constraints. We consider a mechanism with allocation rule

$$
q^{* * k}(\mathbf{x})=\left(\widetilde{S} / S^{*}\right) q^{* k}(\mathbf{x}) \text { for } k \neq k_{0}, \quad q^{* * k_{0}}(\mathbf{x})=1-\sum_{k \neq k_{0}} q^{* * k}(\mathbf{x})
$$

and transfer rule $t_{i}^{* *}(\mathbf{x})=\left(\widetilde{S} / S^{*}\right) t_{i}^{*}(\mathbf{x})$ for $\mathbf{x} \in X$. It is straightforward to check that mecha$\operatorname{nism}\left(\mathbf{q}^{* *}, \mathbf{t}^{* *}\right)$ is DIC and generates the same social surplus as the given BIC mechanism $(\tilde{\mathbf{q}}, \tilde{\mathbf{t}})$. The construction of Theorem 1 then establishes the existence of transfers that together with allocation rule $\mathbf{q}^{* *}$ matches the interim expected utilities $\widetilde{U}_{i}\left(x_{i}\right)$, satisfies DIC constraints, and generates social surplus $\widetilde{S}$.

Theorem 2 provides a sufficient condition for BIC-DIC equivalence for the environments with correlated types. We now present a simple single-unit auction example that illustrate how some BIC mechanisms can generate a smaller social surplus than any DIC mechanism.

Example. Let us consider a single-unit auction example similar to the one discussed in Section 2. We assume that agent types are $x^{1}=1$ and $x^{2}=2$ and that the object has to be assigned to the agents. Hence, the condition of Theorem 2 is not satisfied because there does not exist 
a social alternative where both agents have the smallest value independently of their types. Consider a symmetric allocation rule "allocate to the agent with the smallest value"

$$
\mathbf{q}=\left(\begin{array}{cc}
\frac{1}{2} & 1 \\
0 & \frac{1}{2}
\end{array}\right)
$$

This allocation generates the smallest possible social surplus given that the object has to be assigned to one of the agents. Since allocation $\mathbf{q}$ is not monotone there are no transfers that implement it in dominant strategies (see Mookherjee and Reichelstein, 1992). Therefore, there is no DIC mechanism that generates the smallest social surplus. There exist transfers, however, that implement allocation $\mathbf{q}$ with BIC mechanism. In particular, a symmetric transfer rule

$$
\mathbf{t}=\left(\begin{array}{ll}
0 & -\frac{5}{2} \\
0 & -1
\end{array}\right)
$$

give agents no incentives to deviate from revealing their true values.

\section{Discussion}

In the main text, we present our results for the environments with private values and discrete types to simplify the exposition. The full surplus extraction, however, does not rely on privacy of agent types (see McAfee and Reny, 1992; Chapter 10.2 in Krishna, 2009). In the environments with interdependencies a natural counterpart of dominant strategy incentive compatibility is ex post incentive compatibility (EPIC). Therefore, whenever EPIC mechanisms can generate the social surplus at least as high as any BIC mechanism the results of Theorems 1 and 2 apply. For instance, if agent values satisfy the single crossing condition the generalized VCG mechanism is EPIC and generates the maximum social surplus (see Chapter 10.1 in Krishna, 2009).

If agent values do not satisfy single crossing condition there can exist a BIC mechanism that generates strictly higher social surplus than any EPIC mechanism (see Goeree and Kushnir, 2012, for such an example when agent types are independently distributed). For these environments, however, we establish a partial equivalence: Assume a given BIC mechanism generates the social surplus smaller than the maximum social surplus that can be generated with some EPIC mechanism. Then the construction of Theorem 1 still provides an EPIC mechanism that delivers the same interim expected utilities to all agents and generates at least the same social surplus.

We finally note that our results can be extended to the environments with continuous types using the techniques of McAfee et al. (1989) and McAfee and Reny (1992). 


\section{References}

Anderson, R. and Zame, W. (2001): "Genericity with Infinite Many Parameters," Advances in Theoretical Economics, 1, 1-62.

Barelli, P. (2009): "On the Genericity of Full Surplus Extraction in Mechanism Design," Journal of Economic Theory, 144, 1320-1332.

Bergemann, D. and Morris, S.: (2012) Robust Mechanism Design: The Role of Private Information and Higher Order Beliefs. World Scientific Publishing.

Che, Y.-K. and Kim, J. (2006): "Robustly Collusion-Proof Mechanisms," Econometrica, 74, 1063-1107.

Chen, Y.-C. and Xiong, S. (2013): "Genericity and Robustness of Full Surplus Extraction," Econometrica, 81, 825-847.

Crémer, J. and McLean. R. P. (1985): "Optimal Selling Strategies under Uncertainty for a Discriminating Monopolist when Demands are Interdependent," Econometrica, 53, 345361.

Crémer, J. and McLean. R. P. (1988): "Full Extraction of the Surplus in Bayesian and Dominant Strategy Auctions," Econometrica, 56, 1247-1258.

Csapó, G. and Mller, R. (2012): "Optimal mechanism design for the private supply of a public good," Unpublished manuscript, Maastricht University.

Gizatulina, A., and Hellwig, M. (2010): "Informational Smallness and the Scope for Limiting Informational Rents," Journal of Economic Theory, 145, 2260-2281.

Gizatulina, A., and Hellwig, M. (2012): "Beliefs, Payoffs, Information: On the Robustness of BDP Property in Models with Endogenous Beliefs," Unpublished Manuscript, Max Planck Institute for Research on Collective Goods.

Gershkov, A., Goeree, J. K., Kushnir, A., Moldovanu, B. and Shi, X. (2013): "On the Equivalence of Bayesian and Dominant Strategy Implementation," Econometrica, 81, 197-220.

Goeree, J. K. and Kushnir, A. (2012): "A Geometric Approach to Mechanism Design," Unpublished Manuscript, University of Zürich.

Green, J. and Laffont, J.-J. (1977): "Characterization of Satisfactory Mechanisms for the Revelation of Preferences for Public Goods," Econometrica, 45, 427-438.

Heifetz, A. and Neeman, Z. (2006): "On the Generic (Im)Possibility of Full Surplus Extraction in Mechanism Design," Econometrica, 74, 213-234.

Krishna, V (2009): Auction theory. Academic press.

Laffont, J.-J., and Martimort, D. (2000): "Mechanism Design with Collusion and Correlation," Econometrica, 68, 309-342.

Manelli, A. M. and Vincent, D. R. (2010): "Bayesian and Dominant-Strategy Implementation in the Independent Private Values Model," Econometrica, 78, 1905-1938. 
McAfee, P., McMillan, J. and Reny, P. (1989): "Extracting the Surplus in a Common Value Auction," Econometrica, 57, 14511460.

McAfee, R. and Reny, P. (1992): "Correlated Information and Mechanism Design," Econometrica, 60, 395-421.

Milgrom, P.(2004): Putting auction theory to work. Cambridge University Press.

Mookherjee, D. and Reichelstein, S. (1992): "Dominant Strategy Implementation of Bayesian Incentive Compatible Allocation Rules," Journal of Economic Theory, 56, 378-399.

Papadimitriou, C. and Pierrakos, G. (2011): "On optimal single-item auctions," Proceedings of the 43rd annual ACM symposium on Theory of computing, New York, USA, 119-128.

Peters, M. (2001): "Surplus Extraction and Competition," Review of Economic Studies, 68, 613-631.

Robert, J. (1991): "Continuity in Auction Design," Journal of Economic Theory, 55, 169-179. 